\title{
Curiosity in childhood and adolescence - what can we learn from the brain Matthias J Gruber ${ }^{1,3}$ and Yana Fandakova ${ }^{2,3}$
}

\begin{abstract}
Accumulating evidence in adults has shown that curiosity and surprise enhance memory via activity in the hippocampus, prefrontal cortex, and dopaminergic areas. Based on findings of how these brain areas and their inter-connections develop during childhood and adolescence, we discuss how the effects of curiosity and surprise on memory may develop during childhood and adolescence. We predict that the maturation of brain areas potentially related to curiosity elicitation (hippocampus, anterior cingulate cortex [ACC], prefrontal cortex) and protracted development of hippocampal-PFC and ACC-PFC connectivity lead to differential effects of curiosity and surprise on memory during childhood and adolescence. Our predictions are centred within the PACE (PredictionAppraisal-Curiosity-Exploration) Framework which proposes multiple levels of analyses of how curiosity is elicited and enhances memory.
\end{abstract}

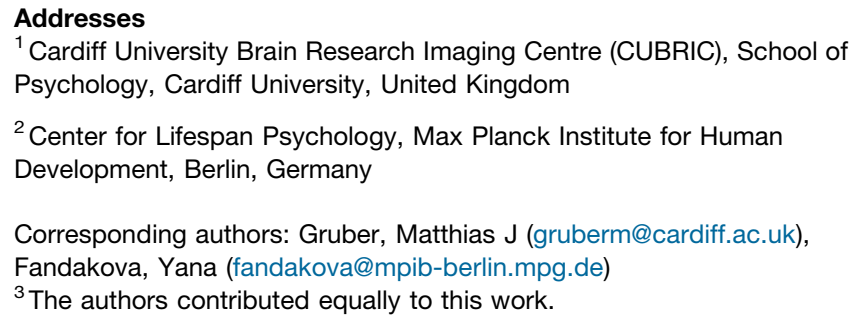
open access article under the CC BY license (http://creativecommons. org/licenses/by/4.0/).

\section{Introduction}

Curiosity, the desire to acquire new information, is often described as an epistemic emotion and is accompanied by positive affect [1]. It has been shown to be a powerful driver of learning, especially in children [2]. In educational settings, curiosity for scientific knowledge is a major motivation for long-term involvement in STEM subjects and predicts academic performance $[3,4]$. Experiencing and expressing higher curiosity during kindergarten predicts academic achievement in primary school, with an even larger influence in children from families with lower socio-economic status $\left[5^{\circ}\right]$. But what are the neural underpinnings underlying the positive effects of curiosity on learning and memory, and how do they develop? Answers to this question would ultimately allow us to design tailored educational approaches to optimally harness how curiosity differently affects learning across development. Furthermore, a neuroscientific approach to study curiosity development offers a unique opportunity to investigate how neural mechanisms underlying learning are modulated by the drive to learn and the satisfaction that comes from learning the desired information.

A plethora of research has consistently demonstrated that infants and young children explore their environment actively in systematic ways, driven by a drive to reduce uncertainty and to close knowledge gaps - both key markers of curiosity $\left[6-8,9^{\circ}\right]$. In addition, the educational literature has emphasized the cognitive and affective mechanisms promoting school-aged children's and adolescents' long-lasting interest and curiosity in such domains as mathematics or physics [10-12]. Yet, we have a limited understanding of how different levels of curiosity affect children's learning because hardly any studies to date have directly measured curiosity or asked children to report on their states of curiosity. Thus, children's (subjective) desire to learn and satisfaction in experiencing desired information has rarely been taken into account when examining curiositybased learning. However, a fledgling line of research in psychology and neuroscience on curiosity in young adults (i.e. 18-30 years of age) has consistently demonstrated how pre-information curiosity, post-information interest, and surprise enhance learning and memory in adults [13-18]. These studies have been employing a trivia paradigm in which participants anticipate answers to general knowledge questions that are associated with varying levels of curiosity about the answer. Using an age-appropriate version of the trivia paradigm, we recently investigated how curiosity and surprise affect memory in children between 10 and 14 years [19 ${ }^{\circ}$. We found that younger children (10-12 years) and adolescents (12-14 years) demonstrated enhanced memory for answers to trivia questions for which they were curious relative to answers to trivia questions about which they were not curious. Furthermore, we found that adolescents - but not children - showed better memory for answers to trivia questions that they judged as more interesting than initially expected. These initial results 
suggest that states of curiosity can indeed be harnessed to facilitate learning in children and adolescents. However, they also point to potential differences in the underlying mechanisms of how positive surprise affects learning across development (Figure 1).

To better understand how curiosity-based learning might develop, we turn to theoretical ideas and current findings in cognitive neuroscience for this opinion piece. In adults, cognitive neuroscience research has started to differentiate the components and neural circuits associated with curiosity-based learning, thereby bridging the fields of memory and motivation $\left[14,15,20,21^{\bullet \bullet}, 22\right]$. Of note, these two rich fields have mostly been studied in isolation, especially in children. We aim to close this gap by integrating recent findings and theoretical ideas on the neural mechanisms of curiosity with findings from developmental cognitive neuroscience to identify candidate mechanisms facilitating the differential effects of curiosity and interest on learning and memory across development.

\section{The Prediction, Appraisal, Curiosity, and Exploration (PACE) Framework and its relationship to child and adolescent development}

An emerging field in neuroscience on curiosity has started to elucidate the neural underpinnings underlying states of curiosity in hon-human primates as well as humans (for reviews, see Refs. $\left[13,21^{\bullet \bullet}, 23^{\bullet \bullet}, 24,25\right]$. Across different experimental manipulations of curiosity (e.g. trivia questions, magic tricks, blurred images, or morbid stimuli), studies in humans have consistently shown that states of curiosity elicit activity in dopaminergic circuit regions, specifically in the ventral striatum $\left[14,15,20,22,26,27,28^{\bullet \bullet}\right]$. In addition, one study has shown that the enhancing effects of curiosity on human long-term memory are supported by activity in the ventral striatum and hippocampus suggesting enhanced hippocampus-dependent memory formation via interactions with the dopaminergic circuit [15] (see also, [22]).

Recently, Gruber and Ranganath proposed a framework that integrates the emergent research on curiosity,

\section{Figure 1}

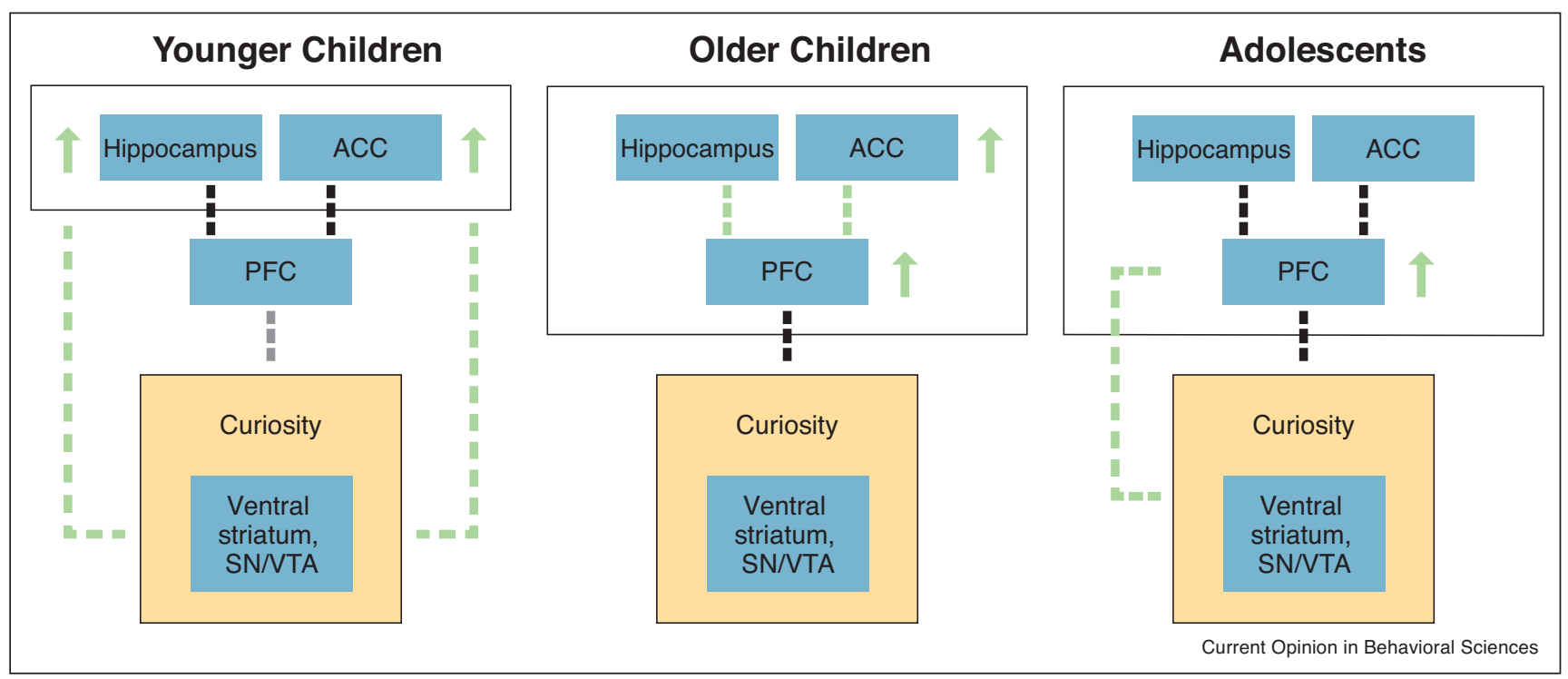

Predictions on the neural correlates of curiosity elicitation during development. Our predictions are centred within the PACE (Prediction, Appraisal, Curiosity, and Exploration) Framework which proposes multiple levels of analyses of how curiosity is elicited and how it enhances memory [21 ${ }^{\circ 0}$ ]. In the PACE Framework, curiosity is triggered by significant prediction errors in the hippocampus and the anterior cingulate cortex (ACC). While prediction errors in the hippocampus are proposed to generally result from encountering novel or unexpected contexts (i.e. contextual prediction errors), prediction errors in the ACC are triggered by cognitive conflict resulting from previous knowledge (i.e. informational prediction errors). PACE suggests that these prediction errors are appraised via lateral prefrontal cortex (PFC) mechanisms in order to evaluate whether the information could be valuable in the future. When curiosity is triggered, a PACE cycle enhances memory encoding through increased attention, exploration, and information-seeking via the dopaminergic circuit, leading to enhanced hippocampus-dependent memory of curiosity-related information. We predict that in young children (left graph) due to ongoing development of the hippocampus and the ACC (indicated by green arrows), hippocampus-dependent and ACC-dependent prediction errors will elicit curiosity in a rather obligatory manner without a strong contribution of PFC-related appraisal processes (indicated by green dashed lines). In older children (middle graph), we expect that age differences in curiosity will primarily result from ongoing development of ACC, PFC along with hippocampal-PFC and ACC-PFC connections (indicated by green arrows and dashed lines, respectively). In adolescents (right graph), protracted PFC development along with connections between the PFC and dopaminergic circuit areas (i.e. ventral striatum and SN/VTA) are proposed as the key mechanisms eliciting curiosity. While brain development in these age groups is not limited to the highlighted regions and their connections, we only depicted those aspects that are proposed to drive corresponding differences in curiosity. 
drawing from a broad range of evidence and theoretical models from psychology and neuroscience on how novelty and prediction errors trigger exploration and information-seeking [21 ${ }^{\bullet \bullet}$ ]. Specifically, Gruber and Ranganath proposed that the effects of curiosity on memory can be understood as emerging from a cycle that involves Prediction errors, Appraisal, Curiosity, and Exploration (PACE). According to this framework, curiosity is first triggered by significant prediction errors, in particular hippocampus-dependent contextual prediction errors and anterior cingulate cortex-dependent informational prediction errors. While prediction errors in the hippocampus are proposed to generally result from encountering novel or unexpected contexts, prediction errors in the anterior cingulate cortex (ACC) depend on cognitive conflict resulting from previous knowledge. PACE suggests that these prediction errors are appraised via lateral prefrontal cortex (PFC) mechanisms as an indicator of information that could be valuable in the future. This cycle enhances memory encoding through increased attention, exploration, and information-seeking via the dopaminergic circuit and enhances hippocampus-dependent memory of curiosity-related information [21 $\left.{ }^{\bullet \bullet}\right]$. Below, we outline how the proposed processes within the PACE framework might help to ultimately better understand the development of curiosity and its effect on memory (Figure 1).

\section{Age differences in context-based and information-based prediction errors}

Hippocampal context-based prediction errors

It has been proposed that the hippocampus forms cognitive maps that allow one to generate predictions based on past experiences with similar contexts and situations [29]. Violations of such generated predictions, in turn, may lead to hippocampal responses that can potentially trigger exploration to resolve this uncertainty and to refine $\operatorname{cog}$ nitive maps [29]. Thus, the hippocampus can be seen as providing the foundation for curiosity through noveltybased or context-based prediction errors that lead to an inherent drive for curiosity-stimulated exploration [21 $\left.{ }^{\circ \bullet}\right]$. Consistent with findings on how the hippocampus supports exploratory eye movements related to prediction errors and novelty [30-32], it has been shown that eye movements related to curiosity predict exploration and attention towards novel information [33,34]. Furthermore, one study investigated individual differences in the strength of one major anatomical pathway connecting the hippocampus with the PFG - the fornix - and its relationship with curiosity [35]. The authors found that individual differences in the microstructure of the fornix predicted specifically diversive curiosity - a curiosity trait that is related to broad exploration triggered by novel events $[35,36]$.

Consistent with these findings in young adults, infants show visual preferences for exploring novel objects, and young children prefer to explore objects if they do not have complete understanding of their functioning $[37,38]$. At the same time, the hippocampus continues to develop in early and middle childhood and supports improvements in memory precision and flexibility [39]. Continued hippocampal maturation may thus contribute to age differences in the ways in which context-based prediction errors stimulate curiosity in younger children. In addition, studies in young adults have shown that surprising information elicits functional connectivity between the hippocampus and the PFC [40-42]. Critically, there are developmental differences in connectivity between the hippocampus and the PFC (e.g. via the fornix or the uncinate fasciculus), which may also contribute to age differences in how hippocampus-mediated prediction errors elicit curiosity. The uncinate fasciculus continues to develop throughout middle childhood [43] and the strength of the uncinate fasciculus microstructure correlates with age-related increases in the ability to modulate attention towards relevant information in children (7-11 years) [44]. Furthermore, a longitudinal study [45] using resting-state functional magnetic resonance imaging found that hippocampus-PFC functional connections only emerged by 13 years of age, suggesting that the transition to adolescence may be an important period for the development of the connections between the hippocampus and the PFC.

Therefore, hippocampal context-based prediction errors may support the computation of unexpected or novel contextual information which may provide the foundation for curiosity in childhood and adolescence. This process may differ from that observed in adults because the relevant functions of the hippocampus and its connections to other subcortical and cortical networks are still developing. While we expect that hippocampal maturation would represent the major source of age differences in hippocampal prediction errors earlier in childhood, changes in hippocampal-PFC connections in the transition to adolescence are expected to make greater contributions to curiosity and its effect on memory later in child development.

\section{Information-related prediction errors in the ACC}

While the hippocampus might compute contrasts in maplike representations elicited by prediction errors, PACE further proposes that the ACC supports the cognitive conflict that is experienced due to information-based prediction errors and information gaps $\left[21^{\bullet \bullet}\right.$. This idea is in line with the theoretical conceptualization of information gaps in terms of cognitive conflict [46] and the neuroscientific literature that has shown enhanced ACC and lateral PFC activity when participants experience cognitive conflict (e.g. Refs. [47,48]), including increased ACG activity during the tip-of-the-tongue experience a phenomenon that has been related to high levels of curiosity $[49,50]$. Consistent with the proposed ACC- 
related cognitive conflict component within the PACE framework, several studies have shown involvement of the ACG when humans and non-human primates await or choose information associated with high curiosity, potentially supporting the idea that the ACC might signal information gaps due to cognitive conflicts that can trigger curiosity $\left[15,22,26,27,28^{\bullet \bullet}, 51\right]$.

During development, age differences in informationrelated prediction errors supported by the ACC may contribute to differences in whether and how such prediction errors stimulate curiosity. While some signatures of cognitive conflict are present even in infancy [52], the ACC continues to mature in childhood and adolescence [53]. In particular, the amplitude of the error-related negativity (ERN), an EEG component associated with the detection and processing of cognitive conflict, increases with age between 8 and 19 years [54]. Thus, protracted development in the neural circuits supporting conflict processing could alter how information-related prediction errors affect the stimulation of curiosity during development. In particular, ACG input associated with conflict monitoring may play an important role in stimulating appraisal-based processes in the lateral PFC. For example, Fandakova and colleagues $\left[55^{\circ}\right]$ found that 8-12 year old children engaged the ACC and anterior insula more strongly during inaccurate and uncertain memory responses, but only $10-12$-year-olds recruited the lateral PFG more strongly for decisions to report uncertainty. Further longitudinal analyses demonstrated that 8-10-year-olds who exhibited greater activation of regions associated with cognitive conflict at a first assessment showed greater increases in PFC activation for uncertain responses 1.5 years later $\left[55^{\circ}\right]$. Consistent with findings of protracted network segregation in childhood [56-58], this initial evidence might suggest that input signals from regions associated with cognitive conflict might contribute to the development of more differentiated appraisal in PFC that would ultimately lead to curiosity. Thus, one hypothesis for future research is that experiencing more information-related prediction errors in a given domain earlier in childhood may contribute to faster development of more efficient PFC-based appraisal in the service of curiosity.

Taken together, after experiencing information gaps due to cognitive conflicts, children and adolescents may become more likely to engage in more differentiated curiosity-driven exploration with increasing age as ACC-based conflict processing improves and contributes to the development of more efficient and differentiated PFC-based appraisal.

\section{Protracted development of appraisal supported by the lateral PFC}

The PACE framework lays out that context-based and information-based prediction errors do not elicit curiosity in an obligatory manner, but that prediction errors are appraised involving lateral PFC functions [21 $\left.{ }^{\bullet \bullet}\right]$ (see also, Refs. $[59,60])$. According to PACE, appraisal of prediction errors can lead to different degrees of curiosity or alternatively to anxiety-related inhibition if one does not have sufficient capability to resolve the uncertainty $\left[21^{\bullet \bullet}, 61\right]$. Consistent with the idea of prefrontal appraisal processes, several neuroimaging studies in young adults have shown lateral PFC activity along with activity in dopaminergic mesolimbic regions when curiosity is elicited $\left[14,15,27,28^{\bullet \bullet}\right]$ (for reviews, see Refs. $\left.\left[21^{\bullet \bullet}, 23^{\bullet \bullet}\right]\right)$. These findings suggest that PFCbased appraisal may be needed to stimulate dopaminergic functions to modulate hippocampus-dependent learning.

Lateral PFC is among the brain regions that shows protracted maturation up to young adulthood [62-64]. Gray matter volume in lateral PFC increases in early childhood, followed by thinning starting around age 910 years and continuing through adolescence [65]. A recent study provided hints that structural changes in the PFC are related to the development of appraisal processes [66]. This longitudinal study examined metamemory development in children between 7 and 15 years. Metamemory — the ability to appraise, self-reflect, and regulate learning and memory outcomes - continued to improve over time aligned to structural changes in the PFC. These findings based on the appraisal of memory retrieval suggest that appraisal processes may develop throughout adolescence, reflecting protracted PFC maturation (see also Ref. $\left[5^{\circ}\right]$ ). Thus, across development curiosity may be elicited to a different degree based on the maturational status of lateral PFC. More specifically, if appraisal processes are still developing in younger children, we expect that (1) they show less lateral PFC modulation by context-based and/or information-based prediction errors and (2) are overall more likely to report higher curiosity rather than differentiating between information associated with high versus low curiosity as older children and adults do. Evolutionarily, there might be an obligatory drive for curiosity in early development or at least an inherent bias towards curiosity over anxiety ( $c f$. Ref. [29]) as prefrontal appraisal processes are still maturing. The protracted development of appraisal processes aligned to PFC maturation may be one neural mechanism enabling an extended exploratory childhood period $\left[67^{\circ \bullet}\right]$, in which context-related and information-related prediction errors may trigger curiosity directly. Future research is necessary to test these hypotheses, but they are consistent with observations that younger children are more likely to show greater interest across a variety of different academic domains, whereas older children have fewer, but clearly differentiated domains of interest [68]. On the neural level, our hypotheses are consistent with research demonstrating that the extent to which lateral PFC activity selectively supports task-relevant versus task-irrelevant information increases with age in $8-13$ year-olds [44]. 
Taking a neuroscientific approach to the development of appraisal processes in service of curiosity and learning can offer unique insights into the interactions of processes associated with the drive to learn (related to dopaminergic circuit functions) and learning itself (related to the hippocampus and memory circuits more generally). For example, our findings that showed that information prediction errors enhanced memory in adolescents more strongly than in children $\left[19^{\circ}\right]$ point to an interaction between the developing PFC appraisal processes and dopaminergic neuromodulation of hippocampus-dependent memory. These interactions are particularly prominent in guiding learning in adolescence [6971] and may enhance curiosity-based learning. In younger children in contrast, the satisfaction associated with learning may emerge from direct triggering of curiosity by context-related and information-related prediction errors. These ideas are consistent with postulated changes in the extent to which cognitive and affective components ( $c f$. Ref. [68,72]) drive curiosity-based learning in development. Ultimately, a neuroscientific approach to the development of appraisal processes in curiosity-based learning will offer unique insights into how these processes interact with the drive to learn within the dopaminergic system and hippocampusbased learning and exploration. In addition, future research might eventually point to optimal ways to harness curiosity-based learning across child development.

\section{Conclusion}

The PACE framework offers an excellent starting point for investigating how brain maturation contributes to curiosity and its effects on learning in childhood and adolescence. First, we expect that hippocampus-related and ACG-related prediction errors (i.e. via novelty and information gaps, respectively) and their effects on curiosity-driven exploration underlie age differences during development due to the ongoing development of these structures. Second, based on the different maturational trajectories of the hippocampus and the ACC, we propose that younger children will show differences to adults in hippocampus-related novelty prediction errors and how they stimulate curiosity. In contrast, older children and adolescents are expected to show differences to adults primarily in ACG-related prediction errors due to cognitive conflict. Finally, as the lateral PFG and its connections to hippocampus and ACC continue to develop, we expect more refined PFC-based appraisal for different strengths of prediction errors which parallels the development of more differentiated curiosity profiles on the behavioral level.

\section{Conflict of interest statement}

Nothing declared.

\section{Acknowledgements}

M.J.G. was funded by a Sir Henry Dale Fellowship from Wellcome and the Royal Society (grant reference number: $211201 / Z / 18 / Z$ ). Y.F. was funded by the Max Planck Society and the Jacobs Foundation. We like to thank (names in alphabetical order) Kathrin Eschmann, Simona Ghetti, Charlotte Murphy, and Charan Ranganath for comments on an earlier version of this manuscript. For the purpose of Open Access, the authors have applied a CC BY public copyright licence to any Author Accepted Manuscript version arising from this submission.

\section{References and recommended reading}

Papers of particular interest, published within the period of review, have been highlighted as:

- of special interest

•• of outstanding interest

1. Vogl E, Pekrun R, Murayama K, Loderer K: Surprised-curiousconfused: epistemic emotions and knowledge exploration. Emotion 2020, 20:625-641.

2. Liquin EG, Lombrozo T: Explanation-seeking curiosity in childhood. Curr Opin Behav Sci 2020, 35:14-20.

3. Levrini O, De Ambrosis A, Hemmer S, Laherto A, Malgieri M Pantano O, Tasquier G: Understanding first-year students' curiosity and interest about physics-lessons learned from the HOPE project. Eur J Phys 2016, 38:025701.

4. von Stumm S, Hell B, Chamorro-Premuzic T: The hungry mind: intellectual curiosity is the third pillar of academic performance. Perspect Psychol Sci 2011, 6:574-588.

5. Shah PE, Weeks HM, Richards B, Kaciroti N: Early childhood - curiosity and kindergarten reading and math academic achievement. Pediatr Res 2018, 84:380-386

In a sample of 6200 children, the authors assessed academic achievement in reading math at kindergarten age along with a parent-report behavioral questionnaire measuring curiosity and effortful control. Greater curiosity was associated with greater kindergarten reading and math academic achievement. This association of curiosity with academic achievement was not moderated by effortful control or sex, but was greater for children from families with low compared to high socioeconomic status. The study highlights that fostering curiosity during kindergarten optimizes academic achievement, especially for children with low socioeconomic status.

6. Schulz LE, Bonawitz EB: Serious fun: preschoolers engage in more exploratory play when evidence is confounded. Dev Psychol 2007, 43:1045-1050.

7. Kidd C, Piantadosi ST, Aslin RN: The Goldilocks effect: human infants allocate attention to visual sequences that are neither too simple nor too complex. PLoS One 2012, 7:e36399.

8. Begus K, Gliga T, Southgate V: Infants' preferences for native speakers are associated with an expectation of information. Proc Natl Acad Sci U S A 2016, 113:12397-12402.

9. Leckey S, Selmeczy D, Kazemi A, Johnson EG, Hembacher E,

- Ghetti S: Response latencies and eye gaze provide insight on how toddlers gather evidence under uncertainty. Nat Hum Behav 2020, 4:928-936

In two studies, the authors investigate how toddlers (aged 25 to 32 months, $N=160$ ) exhibit behaviors that suggest judicious responses to states of uncertainty. Toddlers had to identify a target from two partially occluded similar or dissimilar images. Toddlers accumulated evidence more slowly but required less evidence for similar trials compared with dissimilar trials. By analysing eye movements, toddlers took longer to settle on the selected image during inaccurate trials and switched their gaze between response options more frequently during inaccurate trials. Evidence-accumulation correlated positively with the use of uncertainty language.

10. Hidi S, Renninger KA: The four-phase model of interest development. Educ Psychol 2006, 41:111-127.

11. Ann Renninger K, Hidi SE: The Cambridge Handbook of Motivation and Learning. Cambridge University Press; 2019. 
12. Frenzel AC, Goetz T, Pekrun R, Watt HMG: Development of mathematics interest in adolescence: influences of gender, family, and school context. J Res Adolesc 2010, 20:507-537.

13. Gruber MJ, Valji A, Ranganath C: Curiosity and learning. The Cambridge Handbook of Motivation and Learning. 2019 http://dx doi.org/10.1017/9781316823279.018.

14. Kang MJ, Hsu M, Krajbich IM, Loewenstein G, McClure SM, Wang JT-Y, Camerer CF: The wick in the candle of learning: epistemic curiosity activates reward circuitry and enhances memory. Psychol Sci 2009, 20:963-973.

15. Gruber MJ, Gelman BD, Ranganath C: States of curiosity modulate hippocampus-dependent learning via the dopaminergic circuit. Neuron 2014, 84:486-496.

16. Marvin CB, Shohamy D: Curiosity and reward: valence predicts choice and information prediction errors enhance learning. $J$ Exp Psychol Gen 2016, 145:266-272.

17. Wade S, Kidd C: The role of prior knowledge and curiosity in learning. Psychon Bull Rev 2019, 26:1377-1387.

18. Fastrich GM, Kerr T, Castel AD, Murayama K: The role of interest in memory for trivia questions: an investigation with a largescale database. Motiv Sci 2018, 4:227-250.

19. Fandakova $Y$, Gruber MJ: States of curiosity and interest

- enhance memory differently in adolescents and in children. Dev Sci 2021, 24:e13005

We used a trivia paradigm in which children and adolescents $(N=60,10$ 14 years) encoded trivia questions and answers associated with high or low curiosity. States of high pre-answer curiosity enhanced later memory for trivia answers in both children and adolescents. However, higher positive post-answer interest and surprise enhanced memory for trivia answers beyond the effects of curiosity more strongly in adolescents than in children.

20. Ligneul R, Mermillod M, Morisseau T: From relief to surprise: dual control of epistemic curiosity in the human brain. Neuroimage 2018, 181:490-500.

21. Gruber MJ, Ranganath C: How curiosity enhances

-• hippocampus-dependent memory: the Prediction, Appraisal, Curiosity, and Exploration (PACE) framework. Trends Cogn Sci 2019, 23:1014-1025

The authors propose a neurocognitive framework that integrates the emergent research on curiosity, drawing on a broad range of evidence and theoretical models from psychology and neuroscience on how novelty and prediction errors trigger exploration and information-seeking Specifically, the authors proposed that the effects of curiosity on memory can be understood as emerging from a cycle that involves Prediction errors, Appraisal, Curiosity, and Exploration (PACE). The framework attempts to explain curiosity and memory in terms of cognitive processes, neural circuits, behavior, and subjective experience. The PACE framework generates testable predictions that can stimulate future investigation of the mechanisms underlying curiosity and curiosity-related memory enhancements.

22. Duan H, Fernández G, van Dongen E, Kohn N: The effect of intrinsic and extrinsic motivation on memory formation: insight from behavioral and imaging study. Brain Struct Funct 2020, 225:1561-1574

23. Marvin CB, Tedeschi E, Shohamy D: Curiosity as the impulse to - know: common behavioral and neural mechanisms underlying curiosity and impulsivity. Curr Opin Behav Sci 2020, 35:92-98

The authors discuss the differences and remarkable overlaps underlying curiosity and impulsivity in terms of how they are measured behaviorally and in terms of their underlying neural mechanisms. The authors discuss the growing evidence supporting the importance of frontostriatal circuits and their dopaminergic inputs from the midbrain, areas that are critical to impulsivity and reward processing more generally. The authors proposed how the commonalities between impulsivity and curiosity offer fertile ground for future research into the developmental time course of both constructs and the ways they are influenced by sociocultural context, experimental parameters, neurological conditions, and behavioral interventions.

24. Wang MZ, Hayden BY: Latent learning, cognitive maps, and curiosity. Curr Opin Behav Sci 2021, 38:1-7.

25. Gottlieb J, Oudeyer P-Y: Towards a neuroscience of active sampling and curiosity. Nat Rev Neurosci 2018, 19:758-770.
26. Jepma M, Verdonschot RG, van Steenbergen H, Rombouts SARB, Nieuwenhuis S: Neural mechanisms underlying the induction and relief of perceptual curiosity. Front Behav Neurosci 2012, 6:5.

27. Lau JKL, Ozono H, Kuratomi K, Komiya A, Murayama K: Shared striatal activity in decisions to satisfy curiosity and hunger at the risk of electric shocks. Nat Hum Behav 2020, 4:531-543.

28. Oosterwijk S, Snoek L, Tekoppele J, Engelbert LH, Scholte HS:

-• Choosing to view morbid information involves reward circuitry. Sci Rep 2020, 10:15291

The authors review the emerging literature on curiosity for negative information. The authors propose several factors why people are curious about aversive information. Importantly, these factors might also be important for 'regular' curiosity. The authors' central premise is that curiosity for negative content can be valuable. In particular, negative content may provide an individual with an opportunity to acquire knowledge, reduce uncertainty, experience valued emotions, or engage with the experiences of others. In addition, the authors discuss which factors may motivate or discourage the exploration of negative content.

29. O'keefe J, Nadel L: The Hippocampus as a Cognitive Map. Oxford: Clarendon Press; 1978

30. Voss JL, Bridge DJ, Cohen NJ, Walker JA: A closer look at the hippocampus and memory. Trends Cogn Sci 2017, 21:577-588.

31. Liu Z-X, Shen K, Olsen RK, Ryan JD: Visual sampling predicts hippocampal activity. J Neurosci 2017, 37:599-609.

32. Meister MLR, Buffalo EA: Getting directions from the hippocampus: the neural connection between looking and memory. Neurobiol Learn Mem 2016, 134 Pt A:135-144.

33. Risko EF, Anderson NC, Lanthier S, Kingstone A: Curious eyes: individual differences in personality predict eye movement behavior in scene-viewing. Cognition 2012, 122:86-90.

34. Baranes A, Oudeyer P-Y, Gottlieb J: Eye movements reveal epistemic curiosity in human observers. Vis Res 2015, 117:8190 .

35. Valji A, Priemysheva A, Hodgetts CJ, Costigan AG, Parker GD, Graham KS, Lawrence AD, Gruber MJ: White matter pathways supporting individual differences in epistemic and perceptual curiosity. bioRxiv 2019 http://dx.doi.org/10.1101/642165.

36. Litman JA: Interest and deprivation factors of epistemic curiosity. Pers Individ Differ 2008, 44:1585-1595.

37. Kidd C, Hayden BY: The psychology and neuroscience of curiosity. Neuron 2015, 88:449-460.

38. Schulz L: The origins of inquiry: inductive inference and exploration in early childhood. Trends Cogn Sci 2012, 16:382389.

39. Ghetti S, Fandakova Y: Neural development of memory and metamemory in childhood and adolescence: toward an integrative model of the development of episodic recollection. Annu Rev Dev Psychol 2020, 2:365-388.

40. Gruber MJ, Hsieh L-T, Staresina BP, Elger CE, Fell J, Axmacher N, Ranganath C: Theta phase synchronization between the human hippocampus and prefrontal cortex increases during encoding of unexpected information: a case study. J Cogn Neurosci 2018, 30:1646-1656.

41. Lisman JE, Grace AA: The hippocampal-VTA loop: controlling the entry of information into long-term memory. Neuron 2005 , 46:703-713.

42. Ranganath C, Rainer G: Neural mechanisms for detecting and remembering novel events. Nat Rev Neurosci 2003, 4:193-202.

43. Lebel C, Gee M, Camicioli R, Wieler M, Martin W, Beaulieu C: Diffusion tensor imaging of white matter tract evolution over the lifespan. Neuroimage 2012, 60:340-352.

44. Wendelken C, Lee JK, Pospisil J, Sastre M, Ross JM, Bunge SA Ghetti S: White matter tracts connected to the medial temporal lobe support the development of mnemonic control. Cereb Cortex 2014, 25:2574-2583.

45. Sherman LE, Rudie JD, Pfeifer JH, Masten CL, McNealy K, Dapretto M: Development of the Default Mode and Central 
Executive Networks across early adolescence: a longitudinal study. Dev Cogn Neurosci 2014, 10:148-159.

46. Berlyne DE: Conflict, Arousal, and Curiosity. . McGraw-Hill series in psychology New York, NY, US: McGraw-Hill; 1960.

47. Shenhav A, Botvinick MM, Cohen JD: The expected value of control: an integrative theory of anterior cingulate cortex function. Neuron 2013, 79:217-240.

48. Botvinick MM, Braver TS, Barch DM, Carter CS, Cohen JD: Conflict monitoring and cognitive control. Psychol Rev 2001, 108:624-652.

49. Metcalfe J, Schwartz BL, Bloom PA: The tip-of-the-tongue state and curiosity. Cogn Res Princ Implic 2017, 2:31.

50. Maril A, Wagner AD, Schacter DL: On the tip of the tongue: an event-related fMRI study of semantic retrieval failure and cognitive conflict. Neuron 2001, 31:653-660.

51. Wang MZ, Hayden BY: Curiosity is associated with enhanced tonic firing in dorsal anterior cingulate cortex. bioRxiv 2020 http://dx.doi.org/10.1101/2020.05.25.115139.

52. Goupil L, Kouider S: Behavioral and neural indices of metacognitive sensitivity in preverbal infants. Curr Biol 2016, 26:3038-3045

53. Tamnes CK, Walhovd KB, Torstveit M, Sells VT, Fjell AM: Performance monitoring in children and adolescents: a review of developmental changes in the error-related negativity and brain maturation. Dev Cogn Neurosci 2013, 6:1-13.

54. Overbye K, Walhovd KB, Paus T, Fjell AM, Huster RJ, Tamnes CK: Error processing in the adolescent brain: age-related differences in electrophysiology, behavioral adaptation, and brain morphology. Dev Cogn Neurosci 2019, 38:100665.

55. Fandakova $Y$, Bunge SA, Wendelken C, Desautels $P$, Hunter L,

- Lee JK, Ghetti S: The importance of knowing when you don't remember: neural signaling of retrieval failure predicts memory improvement over time. Cereb Cortex 2018, 28:90-102

The authors used functional MRI to examine how decisions to report uncertainty develop during childhood $(N=119,8-12$ years) and contribute to episodic memory development. The results showed that activity in cingulo-opercular regions during failure to retrieve episodic detail in a source memory task, regardless of whether children were aware that they did not retrieve the target detail, predicted longitudinal increases in PFC activity associated with the decision to report memory uncertainty. Moreover, these activity patterns were associated with greater gains in memory performance over time.

56. Gu S, Satterthwaite TD, Medaglia JD, Yang M, Gur RE, Gur RC, Bassett DS: Emergence of system roles in normative neurodevelopment. Proc Natl Acad Sci U S A 2015, 112:1368113686.

57. DeSerisy M, Ramphal B, Pagliaccio D, Raffanello E, Tau G, Marsh R, Posner J, Margolis AE: Frontoparietal and default mode network connectivity varies with age and intelligence. Dev Cogn Neurosci 2021, 48:100928.

58. Chai LR, Khambhati AN, Ciric R, Moore TM, Gur RC, Gur RE, Satterthwaite TD, Bassett DS: Evolution of brain network dynamics in neurodevelopment. Netw Neurosci 2017, 1:14-30.
59. Silvia PJ: Appraisal components and emotion traits: examining the appraisal basis of trait curiosity. Cogn Emot 2008, 22:94113.

60. Niehoff E, Oosterwijk S: To know, to feel, to share? Exploring the motives that drive curiosity for negative content. Curr Opin Behav Sci 2020, 35:56-61.

61. Ortiz S, Latsko MS, Fouty JL, Dutta S, Adkins JM, Jasnow AM: Anterior cingulate cortex and ventral hippocampal inputs to the basolateral amygdala selectively control generalized fear. J Neurosci 2019, 39:6526-6539.

62. Amso D, Scerif G: The attentive brain: insights from developmental cognitive neuroscience. Nat Rev Neurosci 2015 16:606-619.

63. Crone EA: Executive functions in adolescence: inferences from brain and behavior. Dev Sci 2009, 12:825-830.

64. Zelazo PD, Carlson SM: Hot and cool executive function in childhood and adolescence: development and plasticity. Child Dev Perspect 2012, 47

65. Shaw P, Kabani NJ, Lerch JP, Eckstrand K, Lenroot R, Gogtay N, Greenstein D, Clasen L, Evans A, Rapoport JL et al.: Neurodevelopmental trajectories of the human cerebral cortex. J Neurosci 2008, 28:3586-3594.

66. Fandakova Y, Selmeczy D, Leckey S, Grimm KJ, Wendelken C Bunge SA, Ghetti S: Changes in ventromedial prefrontal and insular cortex support the development of metamemory from childhood into adolescence. Proc Natl Acad Sci U S A 2017. 114:7582-7587.

67. Gopnik A: Childhood as a solution to explore-exploit tensions -• Philos Trans R Soc Lond B Biol Sci 2020, 375:20190502

This article argues that the relatively protracted period of human childhood allows an early period of exploration and presents empirical evidence suggesting that children are highly exploratory in search for information.

68. Frenzel AC, Pekrun R, Dicke A-L, Goetz T: Beyond quantitative decline: conceptual shifts in adolescents' development of interest in mathematics. Dev Psychol 2012, 48:1069-1082.

69. Davidow JY, Foerde K, Galván A, Shohamy D: An upside to reward sensitivity: the hippocampus supports enhanced reinforcement learning in adolescence. Neuron 2016, 92:93-99.

70. van den Bos W, Cohen MX, Kahnt T, Crone EA: Striatum-medial prefrontal cortex connectivity predicts developmental changes in reinforcement learning. Cereb Cortex 2012, 22:1247-1255

71. Hallquist MN, Geier CF, Luna B: Incentives facilitate developmental improvement in inhibitory control by modulating control-related networks. Neuroimage 2018 , 172:369-380.

72. Renninger KA, Ann Renninger K, Hidi SE: Interest development and learning. The Cambridge Handbook of Motivation and Learning. 2019 http://dx.doi.org/10.1017/9781316823279.013. 